\title{
Water-Assisted Laser Drilling for Miniature Internal Thread in Glass and Evaluation of Its Strength
}

\author{
Reo Murakami, Hiroyuki Nakagawa, and Shigeki Matsuo \\ Department of Mechanical Engineering, Shibaura Institute of Technology, \\ 3-7-5 Toyosu, Koto-ku, Tokyo 135-8548, Japan \\ E-mail:matsuos@shibaura-it.ac.jp
}

\begin{abstract}
We report fabrication of miniature internal threads in glass substrates using water-assisted laser drilling, and evaluation of its fracture strength. Miniature internal threads of S0.4 and S0.5 were fabricated using a subnanosecond laser as a light source. The fracture strength of the fabricated internal S0.5 threads was evaluated by applying a load to the commercial male screw that was fitted to the fabricated thread. A model, in which tensile stress causes the fracture, was developed to analyze the fracture phenomenon. The validity of the model was confirmed by the agreement of fracture size between the model and experimental values. Fracture strength of about $1.1 \pm 0.1 \mathrm{MPa}$ was obtained, and this value could be further improved.

DOI: 10.2961/jlmn.2017.03.0005

Keywords: miniature thread, subnanosecond laser, glass, water-assisted laser drilling, fracture strength
\end{abstract}

\section{Introduction}

In the last two decades, interest has grown in material processing with ultrafast lasers. This is because of its applicability for micro/nano processing, non-thermal processing, processing inside transparent materials, and so forth [1-3]. In particular, ultrafast laser processing inside transparent materials has unique capabilities including fabrication of optical waveguide [4] and birefringent device [5], removal processing of three-dimensional (3D) arbitrary shape in micro/submicrometre scale [6-10].

Recently we reported fabrication of S0.5 miniature internal threads in glass substrate using a subnanosecond laser as a light source [11]. Thread is a basic machine element, and miniature threads are useful in joining miniaturized mechanical and electrical parts. However, fabricating miniature threads in brittle materials is difficult. Thus, we adopted water-assisted laser drilling, one of the 3D micro removal processing techniques [7, 12-13]. In the present article we report fabrication of a finer structure of S0.4 miniature internal threads. In addition, we also report evaluation of the fracture strength of the fabricated internal threads. We developed a simple model to explain the shape of the fractured internal thread.

\section{Experimental}

The sample used was mainly glass slides (Matsunami S1111), in addition pyrex and silica glass substrates were also used.

The fabrication and observation procedure of internal miniature screws are already described [11]. Briefly, a 0.5nanosecond Nd:YAG laser (PNP-M08010, Teem Photonics, wavelength of $1,064 \mathrm{~nm}$ ) was used as a light source. Its second harmonic pulses were introduced to an inverted optical microscope (IX-70, Olympus), and focused by a near-infrared objective lens (PLN20x, Olympus, numerical aperture of
0.40 , working distance of $1.2 \mathrm{~mm}$ ). The sample was translated by a three-dimensional motorized stage along the preprogrammed pattern to obtain the desired shape. A little amount of water was dropped on the substrate, and the laser process was carried out from the upper (rear) surface to the lower (front) surface. The fabricated internal threads were observed from the top by optical microscopy. In addition, the substrates were laser-cleaved along the center of the internal thread, and the landscape of the inner surface was observed by optical microscopy.

For the evaluation of the fracture strength, a commercial S0.5 male screw made of stainless steel was fitted to a fabricated internal thread in glass slide, and force was applied to pull out the male screw. S0.5 threads were used because of the availability of male screw. Pulling force was applied by hanging a bucket from the male screw, and filling water in the bucket. For this, the glass slide was placed on a slotted base plate, and the bucket was hung through a slot. The amount of water was gradually increased (increment of about $0.01 \mathrm{~kg}$ ) until the substrate was fractured and the male screw fell down. Totally 10 substrates were tested.

\section{Results and discussion}

\subsection{Fabrication of S0.4 internal threads}

S0.4 miniature internal threads were fabricated in three kind of glass substrates. Figure 1 shows top and cross-sectional views of fabricated S0.4 internal threads in (a) glass slide (thickness: about $1 \mathrm{~mm}$ ), (b) pyrex glass substrate (thickness: about $0.5 \mathrm{~mm}$ ), and (c) silica glass substrate (thickness: about $0.5 \mathrm{~mm}$ ). Spiral pattern can be seen in the inner wall of the through hole. The pitch of the spiral coincided the ISO standard of $0.1 \mathrm{~mm}$. Unfortunately we could not test whether the fabricated internal thread fit to a male screw, because a male screw of S0.4 standard was not available. In the landscape of the inner surface, small chippings 
(a1)

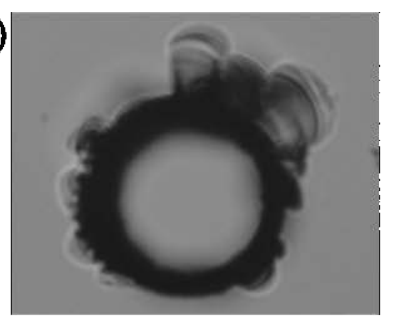

(a2)

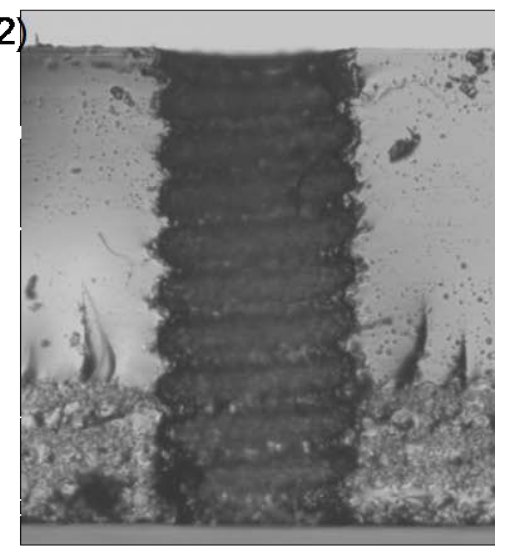

(b)

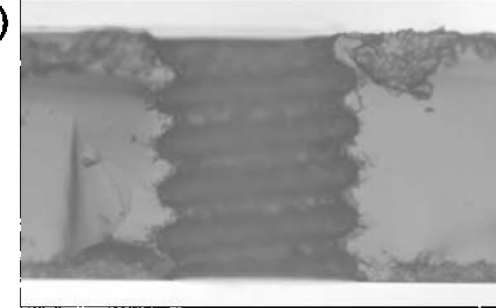

(c)

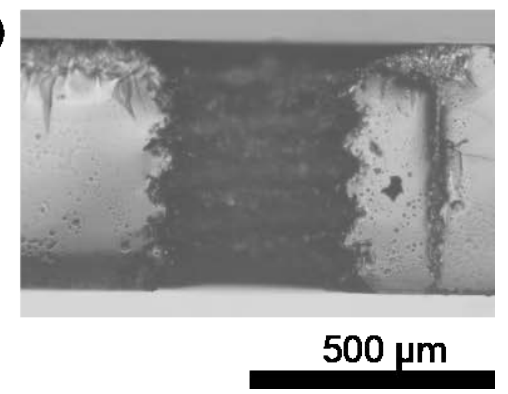

Fig. 1 Top view (a1) and cross-sections (others) of the fabricated S0.4 miniature internal threads. (a) in a glass slide, (b) in a pyrex glass substrate, (c) in a silica glass substrate.

are observed, and the cross sectional profile does not completely much the ISO standard, as observed in the previous article [11]. Use of shorter pulse lasers will decrease such chippings, because undesirable thermal effect will be suppressed [14].

\subsection{Evaluation of fracture strength of the fabricated S0.5 internal threads}

In our test, the fabricated threads could sustain a load of about $2 \mathrm{~kg}$ (about $20 \mathrm{~N}$ ), but by further increasing the load, the substrate was fractured and the male screw fell down. The pattern of fracture was classified into three types. A) formation of a through-hole with a (roughly) circular truncated cone shape around the internal thread, B) segmentation of the substrate into several pieces, and C) A and B occurred simultaneously. Figure 2 shows two examples of fractured
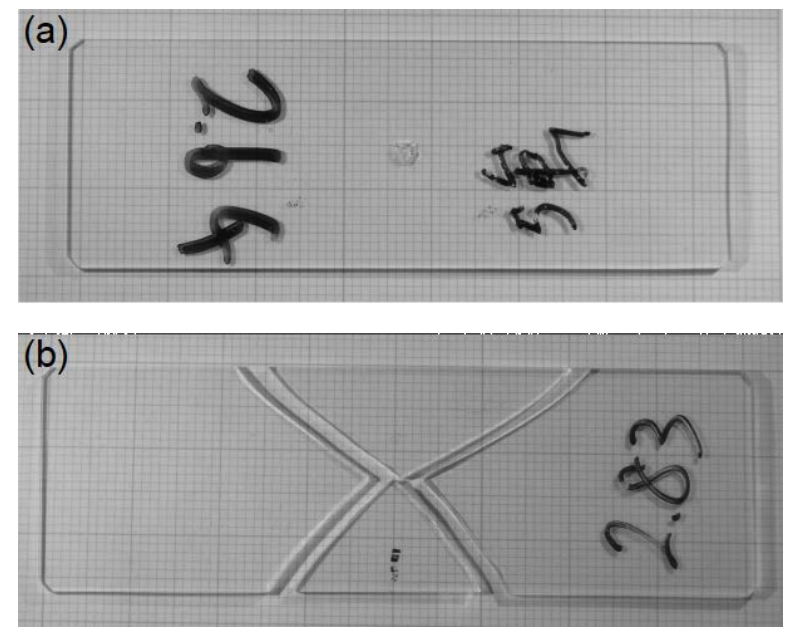

Fig. 2 Examples of fractured substrates. (a) type A fracture, (b) type B fracture. The hand-written numbers indicate the load at the fracture in $\mathrm{kg}$. Below the glass is a $1 \mathrm{~mm}$ grid graph paper.

substrate. The measured fracture strength (load at the fracture) was summarized in Table 1 . As seen, there was no obvious difference in the fracture strength among the types of fracture.

Here we focus on the type A fracture. Three close-up views of type A fracture around the internal thread are shown in Fig. 3. In all of these, the through-hole is not a straight hole but spreading to the bottom ("bottom" indicates the side to which the male screw was pulled). The diameter on the bottom surface was roughly $3 \mathrm{~mm}$, which is much larger than that of the top surface. We analyze the angle of this spreading of the fractured through-hole. In the analysis, we assume that all the phenomena is completely circular symmetric to the center line, and the through-hole has a shape of circular truncated cone. Figure 4 shows the scheme of analysis. Figure 4(a) is the drawing of the cross-section for the analysis. LS is the virtual lateral surface of circular truncated cone with an angle $\theta$. We expect that the fracture occurs along LS at the angle $\theta$ where the tensile stress on LS is maximum.

The force $\boldsymbol{F}_{\text {total }}$ is applied to the male screw in the direction parallel to the center line (perpendicular to the surfaces of the substrate), but in each cross-section, the direction of force exerted on the glass substrate deviates at angle $\beta$, as shown in Fig. 4(b). The angle $\beta$ is determined by the design of the thread (equal to the flank angle). The relation between the forces $\boldsymbol{F}$ and $\boldsymbol{F}_{2}$ is

Table 1 Fracture strength of S0.5 internal screws fabricated in slide glass. A: formation of a throughhole with a (roughly) circular truncated cone shape, B:segmentation of substrate, C: A and B occurred simultaneously.

\begin{tabular}{cccc}
\hline $\begin{array}{c}\text { fracture } \\
\text { type }\end{array}$ & average [N] & $\begin{array}{c}\text { standard error } \\
\text { of the mean [N] }\end{array}$ & number \\
\hline A & $2.3 \times 10^{1}$ & $2.0 \times 10^{0}$ & 5 \\
B & $2.8 \times 10^{1}$ & - & 1 \\
C & $2.5 \times 10^{1}$ & $1.8 \times 10^{0}$ & 4 \\
\hline total & $2.4 \times 10^{1}$ & $2.4 \times 10^{0}$ & 10 \\
\hline
\end{tabular}



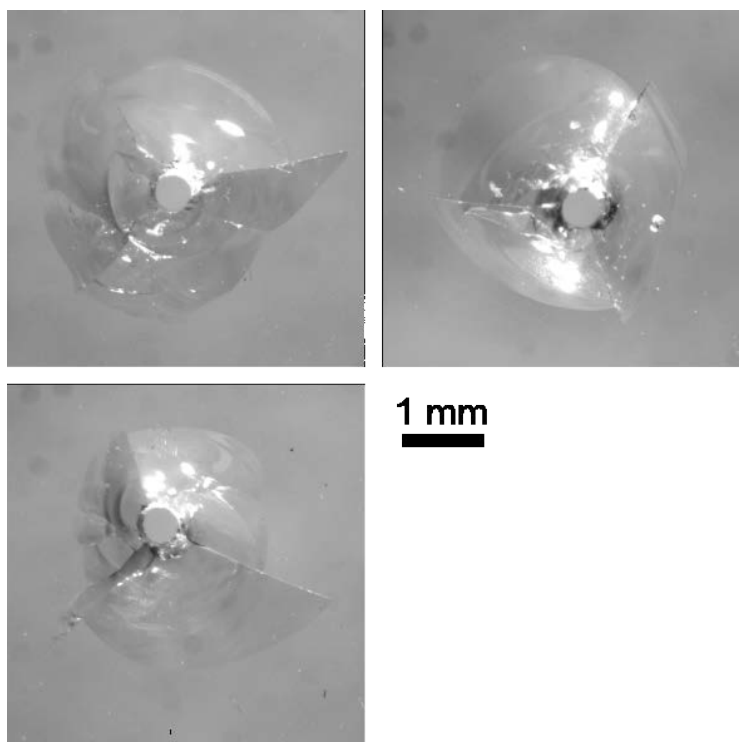

\section{$1 \mathrm{~mm}$}

Fig. 3 Close-up views of type A fracture around the internal thread.

$$
\left|\boldsymbol{F}_{2}\right|=|\boldsymbol{F}| / \cos \beta \text {. }
$$

The tensile force act on the surface LS is

$$
\begin{aligned}
\left|\boldsymbol{F}_{\text {tensile }}\right| & =\left|\boldsymbol{F}_{2}\right| \sin (\theta-\beta) \\
& =|\boldsymbol{F}| \sin (\theta-\beta) / \cos \beta .
\end{aligned}
$$

Here, $\boldsymbol{F}, \boldsymbol{F}_{2}$, and $\boldsymbol{F}_{\text {tensile }}$ are the force in the cross-section, and

$$
\boldsymbol{F}_{\text {total }}=\oint \boldsymbol{F},
$$

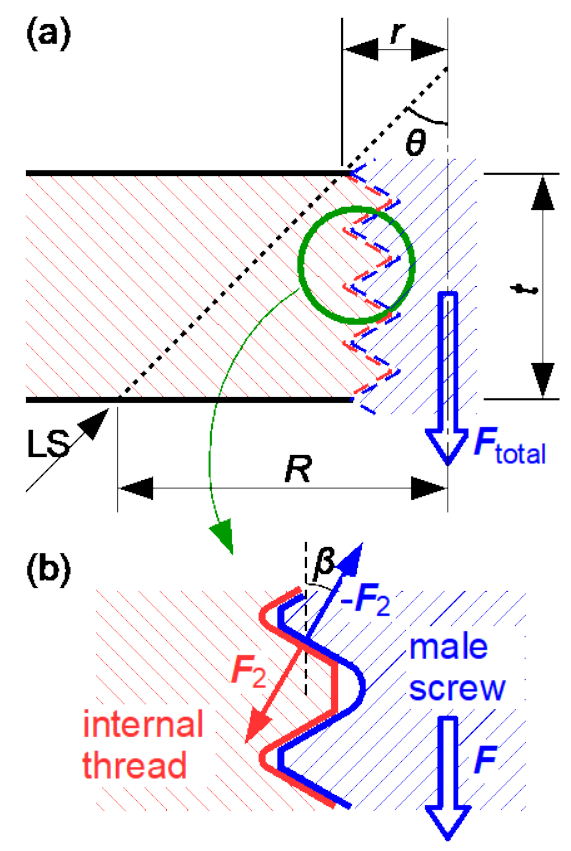

Fig. 4 Scheme of analysis of fracture. (a) Drawing of cross-section of a substrate with miniature internal thread. LS indicates the virtual lateral surface of a circular truncated cone; fracture is expected to occur along LS at the angle $\theta$ where the tensile stress on LS is maximum. (b) Close-up drawing of (a) to show the relation between the forces in a crosssection. $\boldsymbol{F}$ indicates the force exerted to the male screw in this cross-section, and $\boldsymbol{F}_{2}$ and $-\boldsymbol{F}_{2}$ are the force acting between the internal thread and male screw.

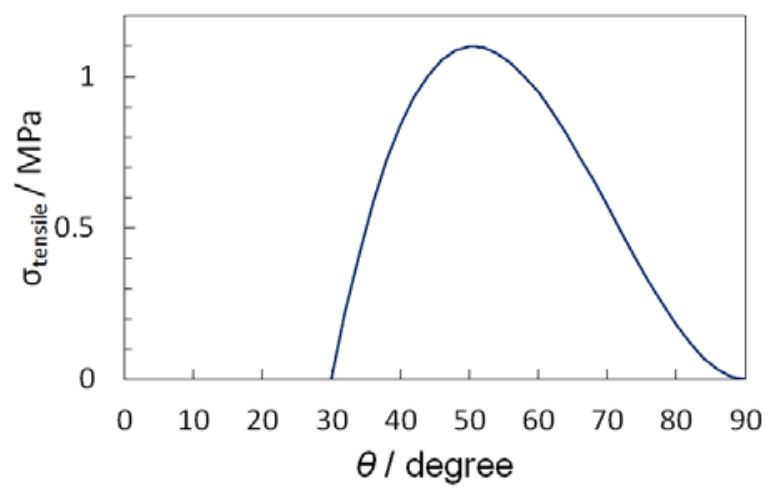

Fig. 5 Calculated $\theta$ dependence of the tensile stress on LS with $t=1 \mathrm{~mm}, r=0.25 \mathrm{~mm}, \beta=30^{\circ}$, and $\boldsymbol{F}_{\text {total }}=2.3 \times 10^{1} \mathrm{~N}$

holds. The area of surface LS is

$$
S_{\mathrm{LS}}=\left(R^{2}-r^{2}\right) \pi /(\sin \theta),
$$

here $r$ is the radius of the upper (top) base, and $R$ is the radius of the lower (bottom) base. The relation

$$
R=r+t \tan \theta,
$$

holds. The tensile stress on the surface LS is represented as

$$
\sigma_{\text {tensile }}=\left\{\left|\boldsymbol{F}_{\text {total }}\right| \sin (\theta-\beta) / \cos \beta\right\} / S_{\mathrm{LS}} \text {. }
$$

Using these equations, $\theta$ dependence of $\sigma_{\text {tensile }}$ is calculated. In the calculation, the values of $t=1 \mathrm{~mm}, r=0.25$ $\mathrm{mm}$ (nominal radius of S0.5 thread), and $\beta=30^{\circ}$ (flank angle) were used. By substituting $\boldsymbol{F}_{\text {total }}=2.3 \times 10^{1} \mathrm{~N}$, we obtain $\theta$-dependence of $\sigma_{\text {tensile }}$ as shown in Fig. 5 . The maximum stress lies around the angle $\theta=50^{\circ}$, which corresponds to the lower diameter $2 R$ of about $2.9 \mathrm{~mm}$. This value approximately agrees the experimentally obtained value. This agreement indicates the validity of this analysis model, although it is a simplified model.

As seen in Fig. 5, the maximum value of tensile stress $\sigma_{\text {tensile }}$ is about $1.1 \mathrm{MPa}( \pm 0.1 \mathrm{MPa}$; the error was estimated as \pm standard error of the mean). This is much smaller than typical fracture strength of glass, 50-100 MPa [15]. This comparison indicates there is plenty of room to improve the strength. Most probably chippings plays a bad role in decreasing the strength. Thus, development of the laser process which does not make chippings, or post-treatment techniques to reduce the chippings, will be helpful to improve the fracture strength (an increase in the fracture strength in precisely-micromachined fused silica without chipping has been shown in literature [16]).

\section{Conclusion}

We fabricated S0.5 and S0.4 miniature internal threads in glass substrates. In addition, the fracture strength of the fabricated threads are evaluated and analyzed. To analyze the fracture phenomenon, we developed a model in which tensile stress on the lateral surface of a circular truncated cone around the internal thread causes the fracture. The analysis showed that the strength is about $1.1 \pm 0.1 \mathrm{MPa}$, and this value could be further improved.

Acknowledgments and Appendixes

This work was supported by the Suzuki Foundation and JSPS KAKENHI Grant Number JP26289019. 


\section{References}

[1] H. Misawa and S. Juodkazis (Eds.): "3D Laser Microfabrication: Principles and Applications” (Wiley-VCH, Weinheim, 2006).

[2] K. Sugioka, M. Meunier, and A. Pique (Eds.): "Laser Precision Microfabrication” (Springer-Verlag, Berlin, 2010).

[3] R. Osellame, G. Cerullo, and R. Ramponi (Eds.): "Femtosecond Laser Micromachining: Photonic and Microfluidic Devices in Transparent Materials" (Springer-Verlag, Berlin, 2012). .

[4] K. M. Davis, K. Miura, N. Sugimoto, and K. Hirao: Opt. Lett., 21, (1996) 1729.

[5] M. Beresna, M. Gecevičius and P. G. Kazansky: Opt. Mater. Express, 1, (2011) 783.

[6] A. Marcinkevičius, S. Juodkazis, M. Watanabe, M. Miwa, S. Matsuo, H. Misawa and J. Nishii: Opt. Lett., 26, (2001) 277.

[7] Y. Li, K. Itoh, W. Watanabe, K. Yamada, D. Kuroda, J. Nishii and Y. Jiang: Opt. Lett., 26, (2001) 1912.

[8] S. Matsuo, S. Kiyama, Y. Shichijo, T. Tomita, S. Hashimoto, Y. Hosokawa, H. Masuhara: Appl. Phys. Lett., 93, (2008) 051107.

[9] J. Gottmann, M. Hermans, N. Repiev, and J. Ortmann: Micromachines, 8, (2017) 110.

[10] C. Hnatovsky, R. S. Taylor, E. Simova, V. R. Bhardwaj, D. M. Rayner, and P. B. Corkum: Opt. Lett., 30, (2005) 1867.

[11] H. Degawa, N. Urano, and S. Matsuo: Micromachines, 8, (2017) 48.

[12] D. J. J. Hwang, T. Y. Y. Choi, and C. P. P. Grigoropoulos: Appl. Phys. A, 79, (2004) 605.

[13] R. An, Y. Li, Y. Dou, D. Liu, H. Yang, and Q. Gong: Appl. Phys. A, 83, (2006) 27.

[14] K. Sugioka and Y. Cheng: Appl. Phys. Rev., 1, (2014) 41303.

[15] The Japan Society of Mechanical Engineers (Ed.): "JSME Mechanical Engineer's Handbook (DVD version)” (The Japan Society of Mechanical Engineers, Tokyo, 2014) p. $\beta 2-175$.

[16] Y. Bellouard: Opt. Mat. Express. 1 (2011) 816.

(Received: June 16, 2017, Accepted: September 30, 2017) 